\title{
Using Syndromic Surveillance to Rapidly Describe the Early Epidemiology of Flakka Use in Florida, June 2014 - August 2015
}

\author{
David Atrubin*, Scott Bowden and Janet J. Hamilton
}

Florida Department of Health, Tampa, FL, USA

\section{Objective}

Objective: To characterize flakka usage in Florida using multiple data sources within the Electronic Surveillance System for the Early Notification of Community-Based Epidemics (ESSENCE-FL)

\section{Introduction}

Syndromic surveillance has historically been used to track infectious disease, but in recent years, many jurisdictions have utilized the systems to conduct all hazards surveillance and provide situational awareness with respect to previously identified issues. Flakka is a synthetic drug (class: cathinones) that recently has been featured in the media. Flakka is a stimulant that causes delusions, aggression, erratic behavior, a racing heart and sometimes death. Two specific counties (one in Florida and one in Kentucky) have been at the center of this emerging epidemic. In August 2015, Florida Department of Health (FDOH) partner agencies requested flakka-related health data in an effort to better understand the epidemiology and context of this problem. ESSENCE-FL is a large syndromic surveillance system, with four main data sources, that captures $87 \%$ of all emergency department (ED) visits statewide.

\section{Methods}

An iterative process was used to design a query to identify flakkarelated visits in the ESSENCE-FL ED data. A concatenated chief complaint - discharge diagnosis field was queried using the following string: $\wedge^{\wedge}$ flakka ${ }^{\wedge}$, or,${ }^{\wedge}$ flaka ${ }^{\wedge}$, or,${ }^{\wedge}$ flacka ${ }^{\wedge}$, or,$\wedge$ flaca ${ }^{\wedge}$, or,${ }^{\wedge}$ flacca ${ }^{\wedge}$. An analysis of the data was conducted to describe flakka-related emergency department visits with respect to person, place, and time. Calls to the Florida Poison Information Center Network (FPICN), one of the four main data sources within ESSENCE-FL, were queried to provide additional situational awareness. Results of the analysis were shared with the Florida Fusion Center and subsequently with law enforcement partners.

\section{Results}

From September 1, 2014 - August 31, 2015, a total of 917 flakkarelated ED visits were identified using the specified query. Figure 1 demonstrates sharply increasing ED visits related to flakka in 2015. In Florida, Broward County was demonstrated to be at the epicenter of this emerging problem. A total of $85 \%$ of all flakka-related visits occurred at Broward County EDs, followed by $10 \%$ at Palm Beach County EDs. Additionally, the analysis showed that males presented to the ED for flakka-related visits nearly four times as frequently as females. Of the 917 flakka-related visits, $80 \%$ of them were in 20-50 year olds. Ninety-one flakka exposure calls were received by the FPICN between June 2015 (when flakka received its own Poison Index Code) and the end of August 2015. These calls demonstrated a nearly identical demographic (66\% Broward County residents, $74 \%$ male, and $72 \%$ of the calls were from the $20-50$ year old age category) as the ESSENCE-FL ED data.

\section{Conclusions}

Having a comprehensive syndromic surveillance system in place that captures nearly all of the ED visits in Florida greatly facilitated this analysis and proved valuable to FDOH's partner agencies. Flakka is an emerging public health and safety issue, which while currently focused in one geographic area of the state, is likely to spread elsewhere. ESSENCE-FL proved useful in rapidly assessing the geographic spread, age, and gender impacted. While syndromic surveillance systems were not initially designed to conduct surveillance for emergent drug usage, having near real-time surveillance systems capable of providing timely, relevant data is critical in quickly characterizing emerging public health issues and helping to prioritize resource utilization. Implementing algorithms that search for previously unused words (e.g., flakka) or words or phrases that are being used in excess of their expected values would be beneficial in detecting these emerging threats more rapidly.

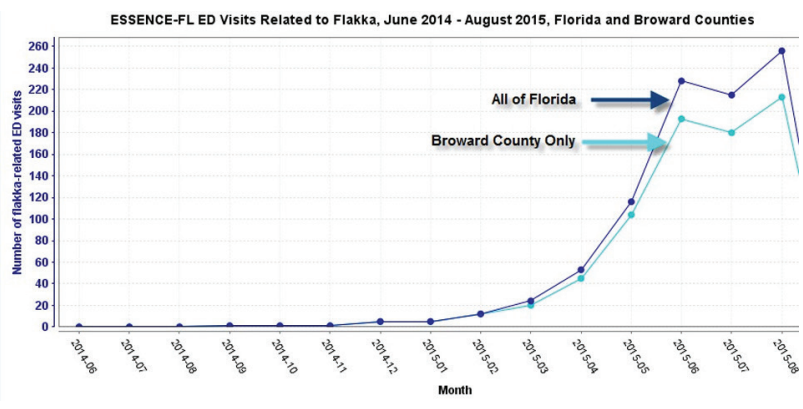

Keywords

flakka; situational awareness; syndromic surveillace; drug use

*David Atrubin

E-mail: david.atrubin@flhealth.gov 